\title{
Fast Universal Spectrophotopolarimeter for Robotic Telescopes
}

\author{
Vladimir Plokhotnichenko, ${ }^{1}$ Grigory Beskin, ${ }^{1}$ Sergey Karpov, ${ }^{1}$ Sergey Bondar, ${ }^{2}$ \\ Vjacheslav de-Boer, ${ }^{1}$ Alexandre Lioubetski, ${ }^{3}$ and Dmitry Badjin ${ }^{4}$ \\ ${ }^{1}$ Special Astrophysical Observatory, Russian Academy of Sciences, Nizhniy Arkhyz 369167, Russia \\ ${ }^{2}$ Institute for Precise Instrumentation, Nizhniy Arkhyz 369167, Russia \\ ${ }^{3}$ VOK Industries, Rostov-on-Don 344000, Russia \\ ${ }^{4}$ Sternberg Astronomical Institute, Moscow State University, Moscow 119992, Russia \\ Correspondence should be addressed to Grigory Beskin, beskin@sao.ru
}

Received 28 June 2009; Revised 10 December 2009; Accepted 10 December 2009

Academic Editor: Alberto J. Castro-Tirado

Copyright ( $) 2010$ Vladimir Plokhotnichenko et al. This is an open access article distributed under the Creative Commons Attribution License, which permits unrestricted use, distribution, and reproduction in any medium, provided the original work is properly cited.

FUSP is the automatic multimode spectrophotopolarimeter aimed to the study of fast optical transients in the photometric, photopolarimetric (instant measurement of linear polarization), and spectropolarimetric (with or without the slit) modes. The mode change is automatic depending on the transient brightness and the purposes of the observations and may be performed in half a second. The instrument may be equipped with either fast EM-CCD or a position-sensitive detector which may provide temporal resolution up to 1 microsecond. FUSP is the robotic instrument for the robotic telescopes.

\section{Introduction}

Until now, the gamma-ray bursts, most energetic events in the Universe, remain mysterious. It is, however, clear that they are generated by a compact relativistic objects such as neutron stars or, more probably, the black holes, either isolated or in binary systems. Internal structure and dynamics of the gamma-ray burst internal engine is inevitably reflected in the temporal structure of the burst emission. Indeed, while the duration of bursts varies in $0.01-$ 100 seconds range, their light curves in $80 \%$ of cases have substructures, while in $66 \%$ the variability time scales are less than 0.1 of total duration of the event [1]. Moreover, light curves of several long bursts display a millisecond temporal structures [2]. At the same time, some models of these events predict the appearance of a significant optical emission along with gamma-ray one, which may reach $8^{\mathrm{m}}$ $12^{\mathrm{m}}[3-5]$. This is especially important as, for a reasonable estimations of a spectral shape, the number of optical photons must be 100-1000 times larger than gamma-ray ones. Unique results of nearly synchronous detection of optical emission from GRB990123 [6] (optical observations started 22 seconds after the burst onset) and completely synchronous from GRB080319B [7] (optical observations performed by a TORTORA [8] wide-field camera with high temporal resolution, which monitored the position of the burst before the event itself) confirm this conclusion. Therefore, the search for, detection of and the detailed, high temporal resolution investigation of optical transients accompanying gamma-ray burst may provide the decisive information on their nature.

It is clear that such observations have to be performed independently from information from space telescopes, as the delays of their triggers may be unacceptably high. They may be replaced by optical monitoring cameras with significant field of view (larger than 100 square degrees), comparable to the ones of satellites. Such cameras may have small, 15-20 cm, objectives, while the information on optical transients they detect may immediately be passed to a larger, $50-200 \mathrm{~cm}$ in diameter, robotic telescope for a detailed investigation which may extract the maximum amount of information on the eventtemporal, photometric, spectral and polarimetric. Such a two-telescope complex has been proposed as a result of development of FAVOR prototype fast wide-field optical camera [9] and is being implemented as a combination of TORTORA wide-field camera and REM robotic telescope [8]. 
Such a complex must satisfy the following set of obvious conditions:

(i) detection of a transient by a wide-field camera in realtime and formation of a pointing trigger for followup instruments in $0.5-1$ second since the onset of event,

(ii) minimal time of distribution of this trigger to one or several robotic telescopes,

(iii) minimal time of repointing of robotic telescope towards the transient (about 1-2 seconds for angular speed of $\left.5-7^{\circ} / \mathrm{s}\right)$,

(iv) maximal temporal resolution of both instrumentsbetter than 0.5 second for wide-field monitoring camera and better than 0.001 second for follow-up instruments,

(v) extraction of maximal amount of information by the follow-up instrument-temporal, spatial, photometric, spectral, and polarimetric one for each detected photon; therefore, this instrument has to be the spectropolarimeter,

(vi) the system must perform completely automatic operation.

Fast Universal SpectroPolarimiter (FUSP) with high temporal resolution is meant to be used on a medium-size telescope as an integral part of a two-telescope complex $[8,10,11]$, or any other telescope for observations with high temporal resolution. The instrument is capable of simultaneous measurements of 3 Stokes parameters of object emission in UBVR bands as well as of objects spectra in a 320-700 nm range, both in slit-less mode and with a spectral slit, with temporal resolution down to 1 microsecond.

Below, we describe the design of this instrument and its use cases inside the two-telescope complex.

\section{Modes of Operation and Optical Scheme of FUSP}

The instrument can perform observations in one of three modes:

(i) photopolarimetry with UBVR filters,

(ii) slitless spectropolarimetry,

(iii) spectropolarimetry with slit.

In the first mode, the photometry and the measurement of linear polarizations in one of UBVR filters are performed for all objects inside $3-5^{\prime}$ instrument field of view. In the second one, four spectra with different orientation of polarization plane are registered for each object, while in the third mode spectra are acquired for the object on the slit only (see Figure 1).

The instrument optical scheme is presented in Figure 2.

The $2^{\prime \prime} \times 5^{\prime}$ spectral slit is placed in the telescope focal plane, and may be replaced with the field lens to achieve larger field of view. The light reflected from mirrors

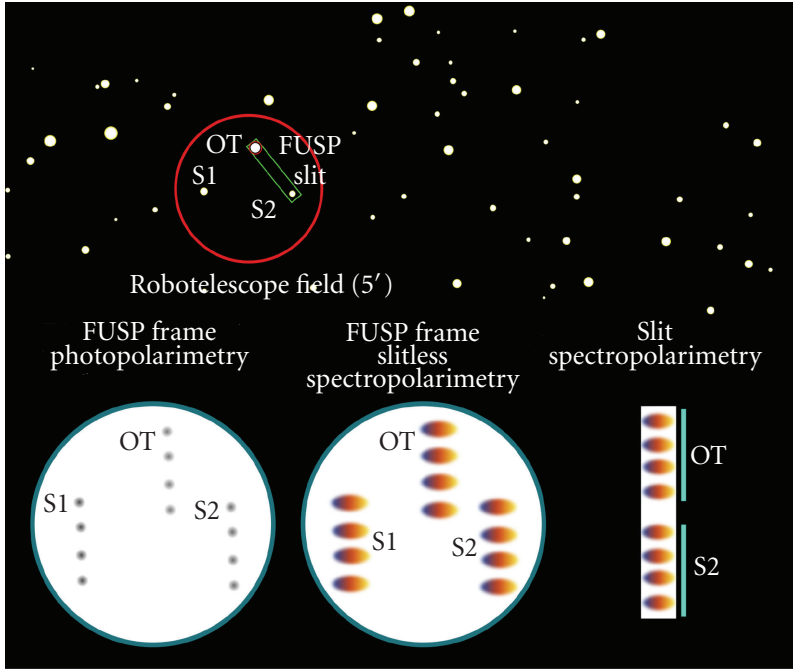

FIGURE 1: Images of FUSP field of view in different operational modes.

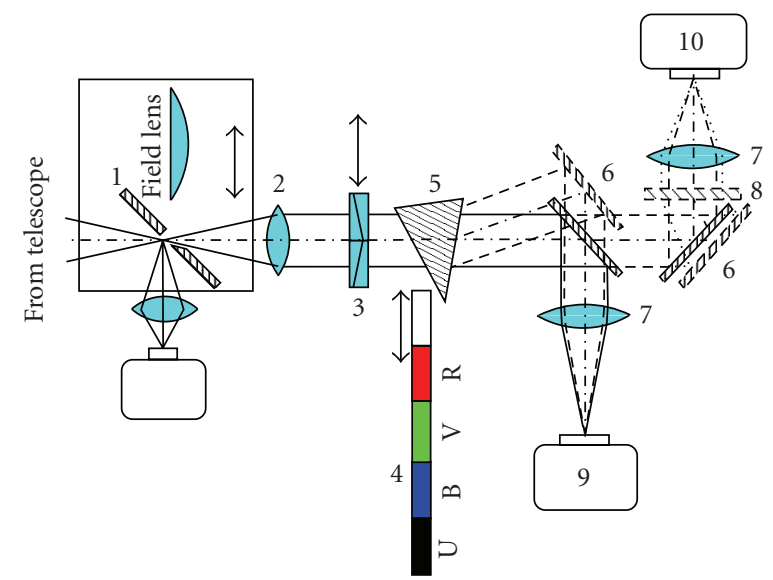

FIGURE 2: Concept of the FUSP. 1: movable mirror with the slit for a transmission of light to TV guiding camera, which may be replaced with the field lens; 2: collimator; 3: double Wollaston prism (polarizer); 4: movable $\mathrm{U}, \mathrm{B}, \mathrm{V}, \mathrm{R}$ and white light filters; 5: movable spectral prism; 6: units of light transmission mirrors, movable synchronously with 5 and 8; 7: objectives; 8: diffraction grating; 9: Position-Sensitive detector; 10: EM-CCD. The field lens is used on a telescopes with large relative apertures to achieve wider field of view without vignetting; for systems with smaller D/F ratio it may be omitted.

(1) on edges of the slit is directed towards the guiding camera, aimed for a precise pointing of telescope towards the object. On-axis light beam passes through the collimator (2) and reaches one of two detectors $(9,10)$. On its way it may be decomposed into spectral (5) and polarimetric (3) components and passed through one of UBVR color filters (4). All necessary components for that are inserted in the parallel on-axis beam automatically after receiving the information on the source type and brightness according to a mode-selection algorithms. 


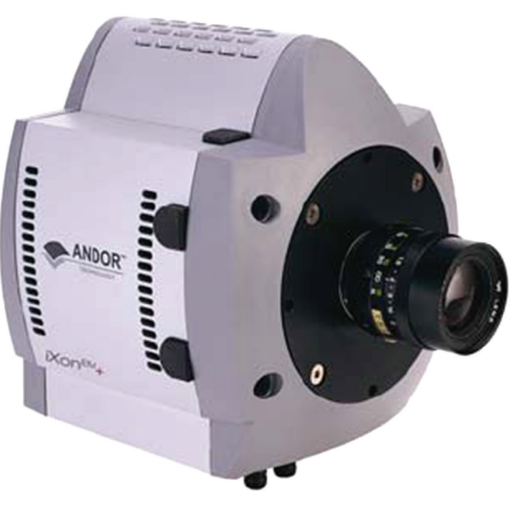

(a)

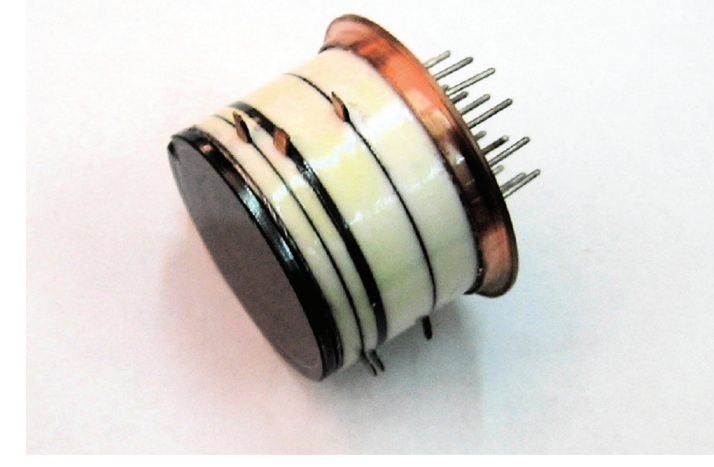

(b)

FIgure 3: Two possible photodetectors which may be used in FUSP. (a) iXon ${ }^{\mathrm{EM}}+888$ EM-CCD from Andor. (b) Position-Sensitive Detector with GaAs-photocathode, developed at Special Astrophysical Observatory of Russian Academy of Sciences.

All optical parts of the instrument are manufactured from the materials with high UV transparency. The objective (7) focal length is 3 times larger than the collimator one, to cover the whole photocathode of PSD with region of interest in focal plane.

Below are details on several components of the FUSP.

Double Wollaston prism (3) is used in a way similar to one described in [12], to decompose the initial parallel light beam into four ones corresponding to polarization planes separated by $45^{\circ}$. Photometry of four corresponding object images gives three Stokes parameters, that is, linear polarization.

Units of light transmission mirrors (6) allow to equip the instrument with two photodetectors, EM-CCD (10) and Position-Sensitive Detector (9), simultaneously, and use any of them in either imaging or spectral mode, by inserting or removing corresponding units $(4,5)$. The objective $(7)$ parameters are selected so that the field of view linear size coincides with detector input size. When working in spectral mode, with either prism or diffraction grating, corresponding mirror (6) positions are selected.

\section{FUSP Detectors}

$\mathrm{EM}$ CCD $\mathrm{iXon}^{\mathrm{EM}}+888$ is an $1 \mathrm{k} \times 1 \mathrm{k}$ back-illuminated EMCCD produced by Andor [13], and has a single photon detection capability without an image intensifier. The quantum efficiency is up to $90 \%$. The operational frame rates are from 8.9 per second (full frame) till 310 per second $(8 \times 8$ binning with $128 \times 128$ pixels work area). The pixel size is $13 \mu \mathrm{m}$ (see Figure 3).

Position-Sensitive Detector, developed at Special Astrophysical Observatory of Russian Academy of Sciences, is a vacuum tube with multialkali [14] or GaAs [15] photocathode and microchannel plates to multiply the electron avalanches. Coordinates of incoming photons are measured by means of multisection metallic collector anode. Input area is $18 \mathrm{~mm}$ in diameter, with $20-70 \mu \mathrm{m}$ resulting resolution element. Quantum efficiency is up to $\sim 30 \%$ for GaAs and up to $\sim 15 \%$ for multialkali photocathode, and acquisition dead time is about 1 microsecond. The accuracy of photon arrival time measurement is also 1 microsecond, with limiting detection rate of $\sim 10^{5}$ counts per second from the whole photocathode (see Figure 3).

The microsecond temporal resolution is achieved by means of "Quantochron 4-48" acquisition system [16]—a fast chronometer intended for measurement and acquisition of characteristics of standard discrete event sequences, which is a further development of a data acquisition system described in $[17,18]$. It is based on a SPARTAN Field Programmable Gate Array, is programmed in VHDL logical matrices design language, and is designed to interface with acquisition PC through the standard PCI bus. The "Quantochron 4-48" is synchronized with GPS receiver and allows to register the photon time of arrivals with 30-nanosecond accuracy; its internal dead time is also 30-nanosecond, and the limiting flux it able to register without data losses is $10^{6}$ counts per second.

\section{Principles of Operation}

Upon receiving of a new source coordinates from either gamma-ray telescope or wide-field monitoring camera, the robotic telescope equipped with FUSP is pointed towards it. Inside the instrument, the light is reflected from the mirror edges of the slit towards the TV guide, which may be used to correct the pointing and precisely place the transient onto the slit. Then, if the object is bright enough, the slit opens and the light passes through the optical units towards the photodetectors. If, however, the object is too faint to be localized by TV guide, the slit unit moves out along the slit direction, and the field lens is inserted into the light beam. In this regime, all photons from FUSP field of view sky region may be recorded for a detailed study later. 


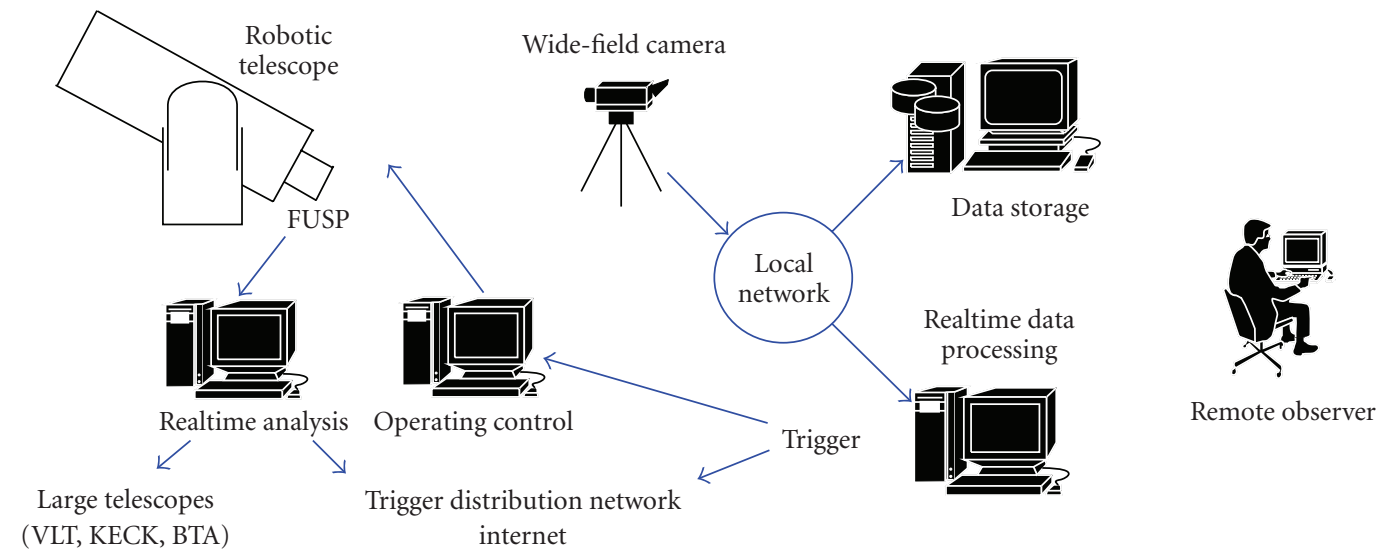

FIGURE 4: General scheme of a two-telescope complex for autonomous detection and investigation of short optical transients. The robotic telescope is equipped with FUSP and is meant to study transients detected by a wide-field monitoring camera in a few seconds after their onset.

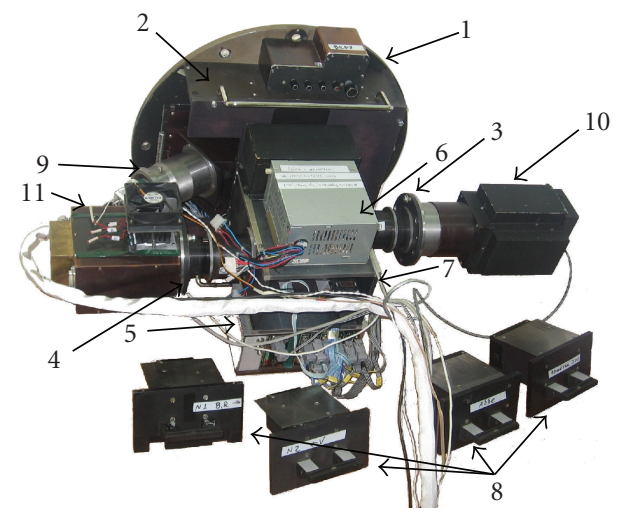

Figure 5: Overview of the Multimode Panoramic PhotoSpectroPolarimeter (MPPP). 1: telescope mounting flange; 2: focal plane unit; 3 and 4: flanges for mounting the Position-Sensitive Detectors or EM-CCDs; 5: instrument state and motors control unit; 6: power supply; 7: mount point for interchangeable light transformation units; 8: set of optical units; 9: TV-CCD guide; 10 and 11: two PSDs, which may be replaced with EM-CCDs.

All the units are configured and inserted into the light beam automatically, according to the brightness of the transient and the program of observations.

\section{Desired Performance}

The FUSP instrument is supposed to be used on a smallaperture robotic telescopes with relative aperture smaller than $1: 4$. The spectral range is defined mainly by the type of detector used-for PSD it is from 350 till $700 \mathrm{~nm}$, while for typical CCD it is $400-700 \mathrm{~nm}$. The spectral resolution may vary from 10 to 100 and is defined by the telescope focal length. Temporal resolution is 1 microsecond for PSD photon counter and is down to $1 \mathrm{~ms}$ for fast CCDs.

For a typical $60 \mathrm{~cm}$ telescope, in photopolarimetric mode the instrument is able to measure $10 \%$ linear polarization
(3 Stokes parameters simultaneously) at a $5 \sigma$ level (S/N $=5$, polarization measurement accuracy of about $2 \%$ ) for a $\mathrm{V} \approx 13.5^{\mathrm{m}}$ object using 1-second exposure. In slitless spectropolarimetric mode under moderate seeing and sky brightness conditions it is possible to measure a $10 \%-15 \%$ linear polarization at a level of $\mathrm{S} / \mathrm{N}=5$ for an object with $\mathrm{V} \approx 11.5^{\mathrm{m}}-12^{\mathrm{m}}$ in 1 second. For a spectropolarimetry with slit the accuracy of polarization measurements for 1-second exposure is about $2 \%$ for $\mathrm{V} \approx 11.5^{\mathrm{m}}-12^{\mathrm{m}}$ objects.

\section{FUSP inside the Two-Telescope Complex}

The FUSP instrument may, in principle, be used alone, for the investigation of objects with known positions, such as black hole candidates, flaring stars, and pulsars and so forth. For gamma-ray bursts, however, the positions are not known in advance; so the instrument must be accompanied by the wide-field monitoring camera, able to perform continuous monitoring of large sky regions, to automatically detect and perform preliminary classification of optical transients, and to pass their positions to FUSP-equipped telescope. Scheme of such two-telescope complex is shown in Figure 4.

The wide-field monitoring camera performs the realtime analysis of its data, detects the appearance of new transients or flux change of previously visible objects, performs the basic classification of such events and, if the transient looks like the flash from GRB, passes its information to robotic telescope equipped with FUSP. Control software then initiates the repointing of robotic telescope and, while it is moving, decides on an optimal mode of operation of the instrument depending on transient brightness, and reconfigures the FUSP accordingly.

\section{Current Status of the Instrument}

We already built and presently use in observations on a Russian 6-m telescope the prototype of FUSP-the Multimode Panoramic PhotoSpectroPolarimeter (MPPP) [19]. 


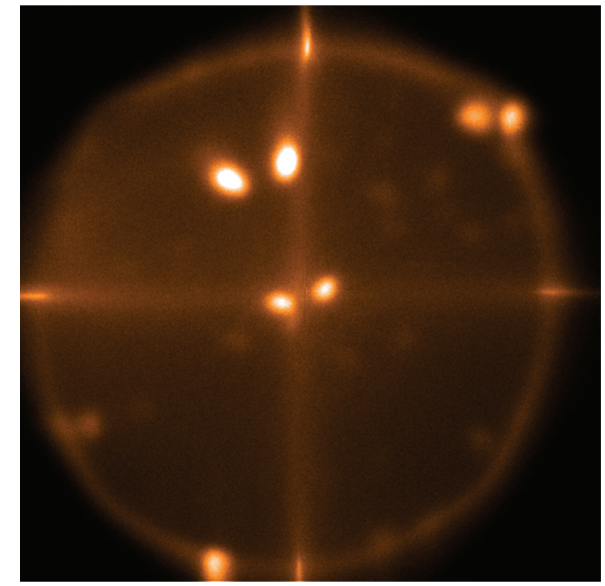

(a)

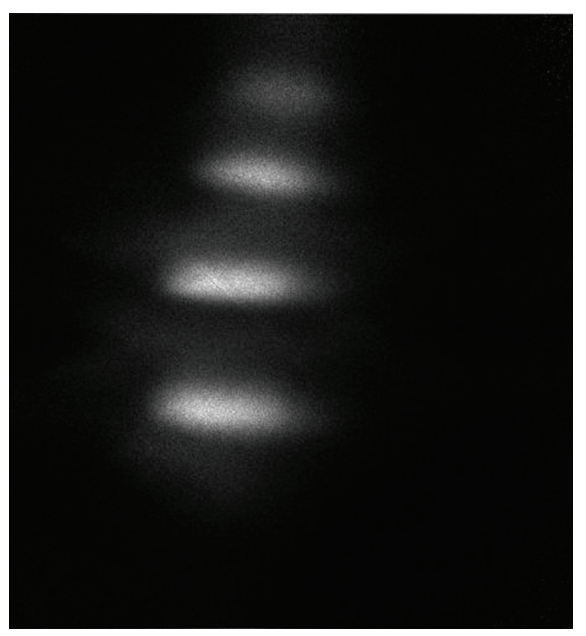

(c)

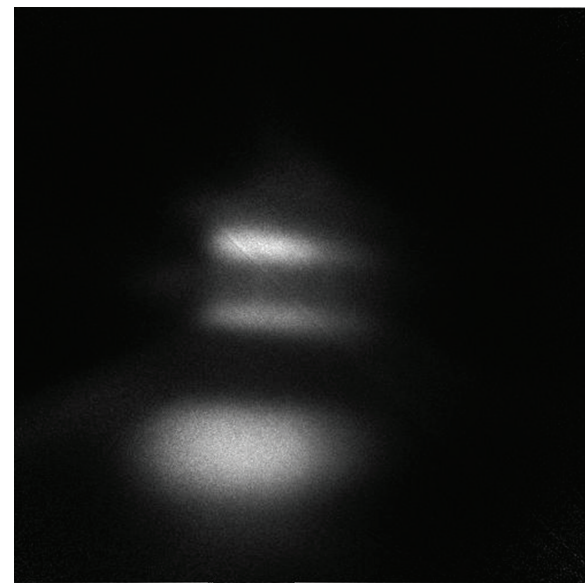

(b)

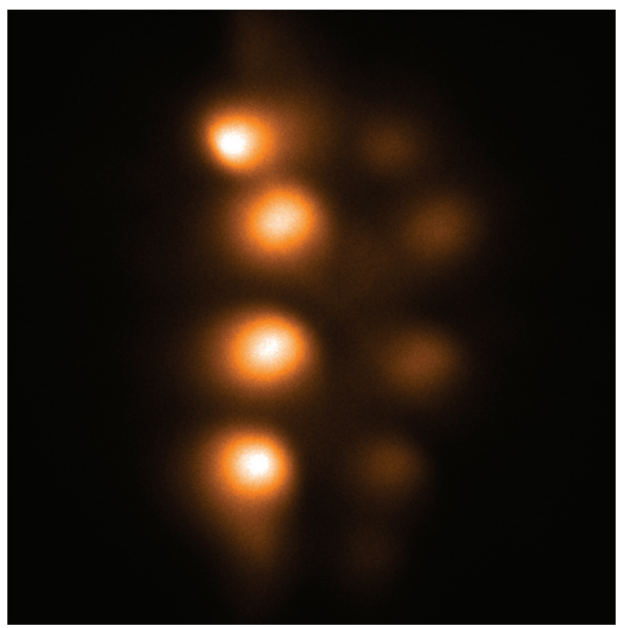

(d)

Figure 6: The different modes of MPPP operation. (a) One-color photopolarimetric mode with two images of each object in wide field. (b) Spectroscopic mode with low-resolution spectrum of object, along with ones of comparison star and the region of the sky used for calibration. (c) Spectropolarimetric with object spectra in four different polarizations. (d) Multicolor photopolarimetric mode with four images of object in two colors.

The main difference of this instrument is what it is purposed for a preplanned observations, and so it lacks the remotely controlled mode change capability. The needed optical unit is inserted into the light beam before the observations manually. This instrument with the set of optical units, providing different modes of operation is shown in Figure 5. Example images of objects observed with instrument in different regimes are shown in Figure 6.

\section{Acknowledgments}

This work was supported by the Bologna University Progetti Pluriennali 2003, by Grants of CRDF (no. RP1-2394-MO02), RFBR (no. 04-02-17555, 06-02-08313, and 09-0212053-ofi-m), INTAS (04-78-7366), and by the Presidium of the Russian Academy of Sciences Program. SoKarpor has also been supported by grant of the President of Russian Federation for federal support of young scientists.

\section{References}

[1] T. Piran, "The physics of gamma-ray bursts," Reviews of Modern Physics, vol. 76, no. 4, pp. 1143-1210, 2005.

[2] S. McBreen, F. Quilligan, B. McBreen, L. Hanlon, and D. Watson, "Temporal properties of the short gamma-ray bursts," Astronomy \& Astrophysics, vol. 380, no. 2, pp. L31-L34, 2001.

[3] D. Eichler and G. Beskin, "Nonthermal optical transients from relativistic fireballs," Physical Review Letters, vol. 85, no. 13, pp. 2669-2672, 2000.

[4] E. P. Liang, A. Crider, M. Böttcher, and I. A. Smith, "GRB 990123: the case for saturated comptonization," Astrophysical Journal, vol. 519, no. 1, pp. L21-L24, 1999.

[5] X. F. Wu, Z. G. Dai, Y. F. Huang, and T. Lu, "Optical flashes and very early afterglows in wind environments," Monthly Notices of the Royal Astronomical Society, vol. 342, no. 4, pp. 1131$1138,2003$.

[6] C. Akerlof, R. Balsano, S. Barthelmy, et al., "Observation of contemporaneous optical radiation from a $\gamma$-ray burst," Nature, vol. 398, no. 6726, pp. 400-402, 1999. 
[7] J. L. Racusin, S. V. Karpov, M. Sokolowski, et al., "Broadband observations of the naked-eye $\gamma$-ray burst GRB 080319B," Nature, vol. 455, no. 7210, pp. 183-188, 2008.

[8] E. Molinari, S. Bondar, S. Karpov, et al., "TORTOREM: twotelescope complex for detection and investigation of optical transients," Nuovo Cimento della Societa Italiana di Fisica B, vol. 121, no. 12, pp. 1525-1526, 2006.

[9] S. Karpov, G. Beskin, A. Biryukov, et al., "Optical camera with high temporal resolution to search for transients in the wide field," Nuovo Cimento della Societa Italiana di Fisica C, vol. 28, no. 4-5, pp. 747-750, 2005.

[10] S. Karpov, D. Bad'in, G. Beskin, et al., "FAVOR (FAst Variability Optical Registration)—two-telescope complex for detection and investigation of short optical transients," Astronomische Nachrichten, vol. 325, no. 6-8, p. 677, 2004.

[11] G. Beskin, V. Bad'in, A. Biryukov, et al., "FAVOR (FAst Variability Optical Registration) - a two-telescope complex for detection and investigation of short optical transients," Nuovo Cimento della Societa Italiana di Fisica C, vol. 28, no. 4-5, pp. 751-754, 2005.

[12] E. Oliva, "Wedged double Wollaston, a device for single shot polarimetric measurements," Astronomy and Astrophysics Supplement Series, vol. 123, no. 3, pp. 589-592, 1997.

[13] "Andor Technology," http://www.andor.com.

[14] V. Debur, T. Arkhipova, G. Beskin, et al., "Position-sensitive detector for the 6-m optical telescope," Nuclear Instruments and Methods in Physics Research, Section A, vol. 513, no. 1-2, pp. 127-131, 2003.

[15] V. de-Bur, A. Terekhov, S. Kosolobov, et al., "Position-sensitive detector with GaAs photocathode and high time resolution," in Time Resolution Astrophysics: The Universe at Sub-Second Timescales, D. Phelan, O. Ryan, and A. Shearer, Eds., vol. 984 of American Institute of Physics Conference Series, pp. 186-193, 2008.

[16] V. L. Plokhotnichenko, A. V. Solin, and A. G. Tikhonov, "The "Quantochron 4-48" facility for registration of random fluxes of multidimensional signals," Astrophysical Bulletin, vol. 64, no. 2, pp. 204-212, 2009.

[17] A. V. Zhuravkov, A. A. Pimonov, and V. L. Plokhotnichenko, "“QUANTOCHRON"-a multichannel time-to-code converter," Bulletin of the Crimean Astrophysical Observatory, vol. 37, pp. 159-172, 1994.

[18] V. Plokhotnichenko, G. Beskin, V. Debur, A. Panferov, and A. Panferova, "The multicolor panoramic photometerpolarimeter with high time resolution based on the PSD," Nuclear Instruments and Methods in Physics Research, Section $A$, vol. 513, no. 1-2, pp. 167-171, 2003.

[19] V. Plokhotnichenko, G. Beskin, V. de-Bur, et al., "Devices and software for optical panoramic observations with microsecond time resolution," in High Time Resolution Astrophysics: The Universe at Sub-Second Timescales, D. Phelan, O. Ryan, and A. Shearer, Eds., vol. 984 of American Institute of Physics Conference Series, pp. 194-201, 2008. 

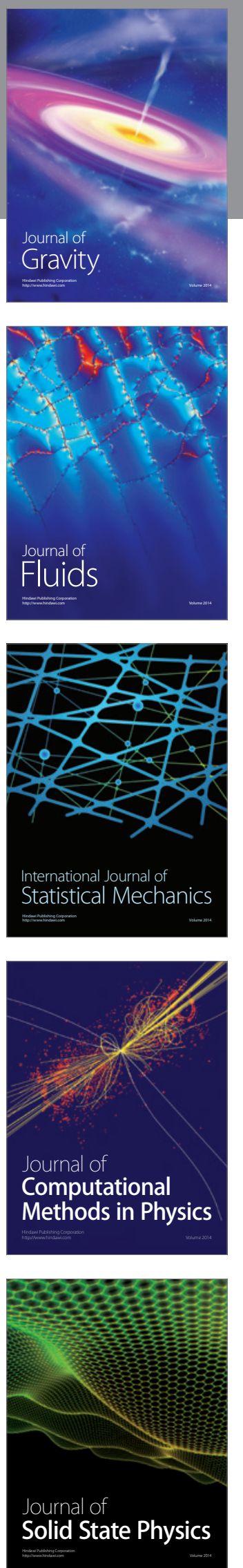

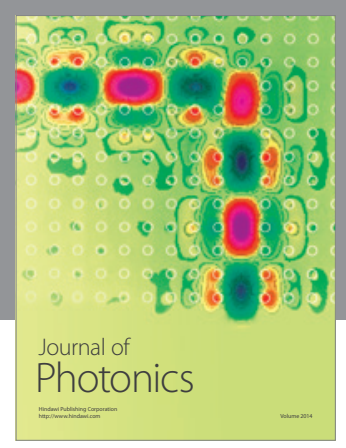

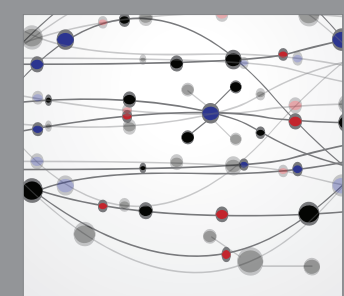

The Scientific World Journal
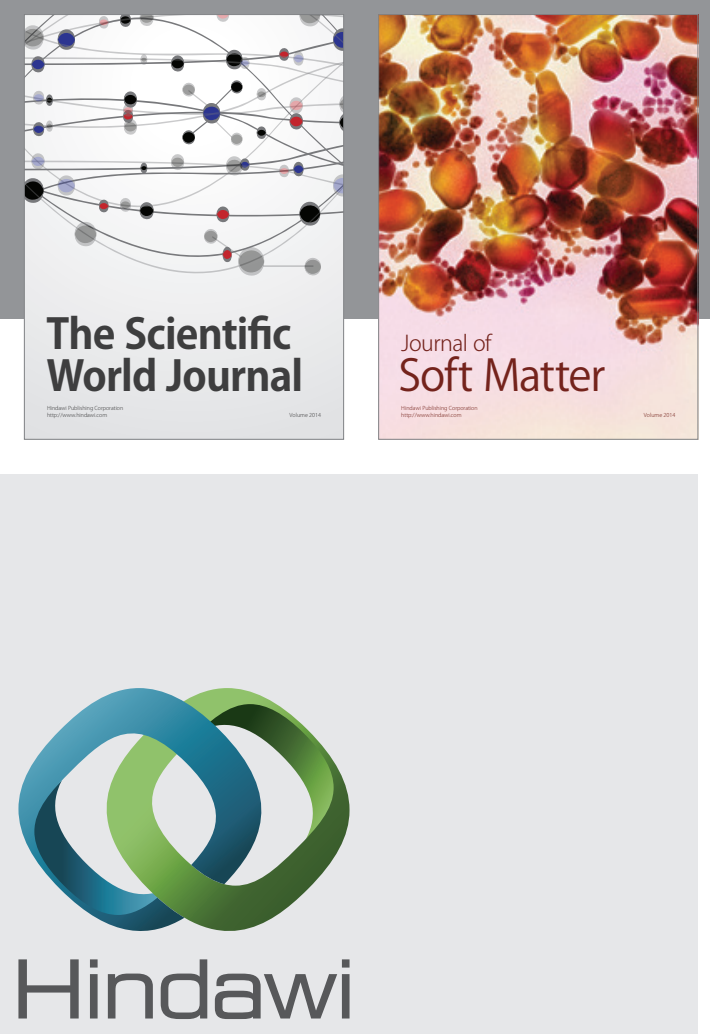

Submit your manuscripts at

http://www.hindawi.com
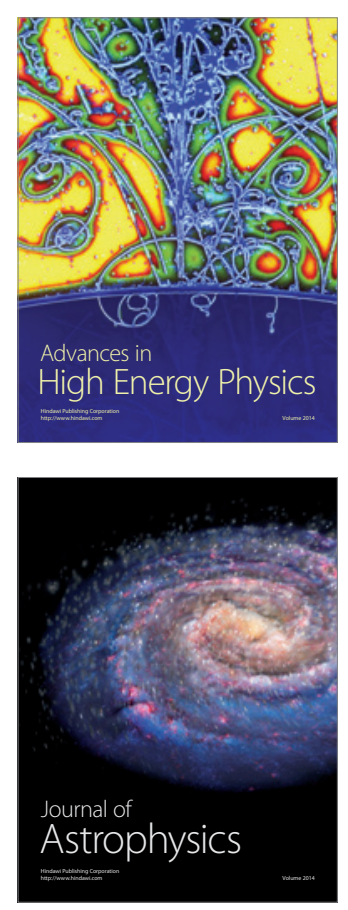
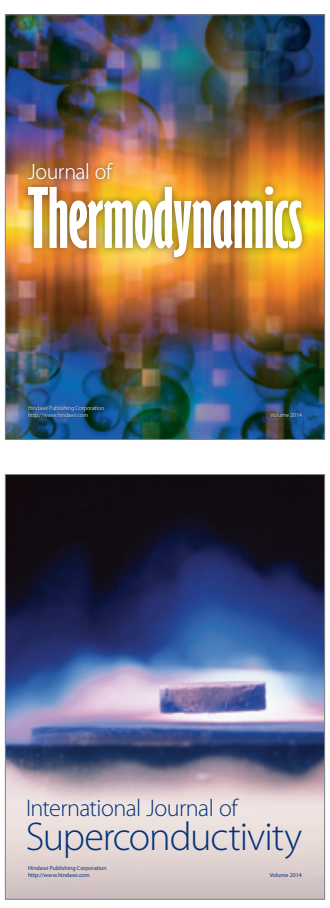
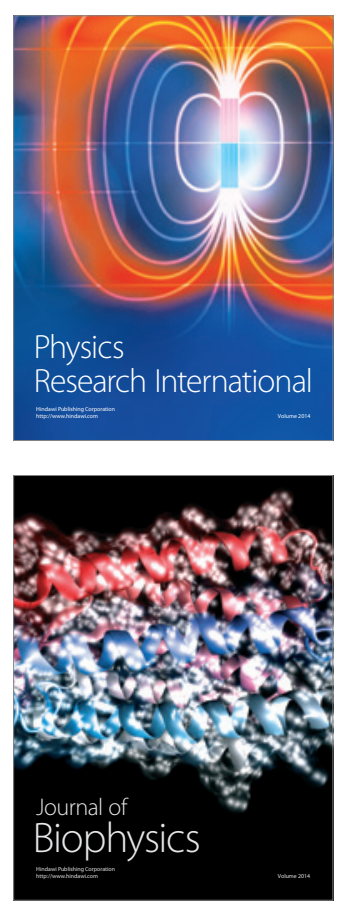
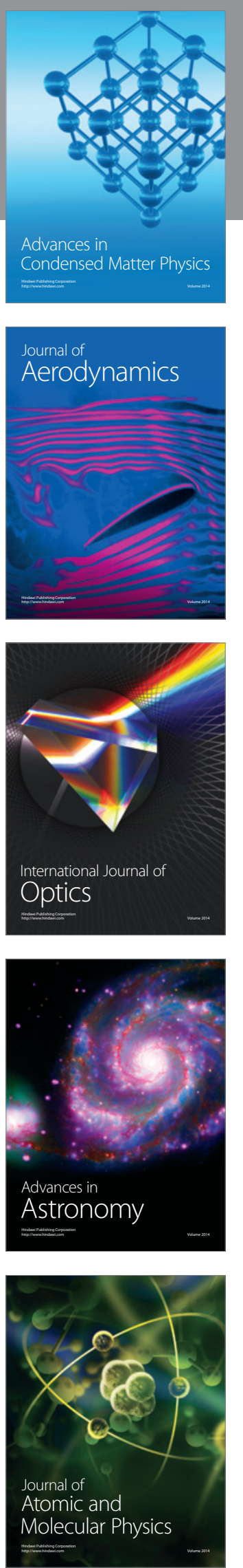